\title{
Burden of disease and change in practice in critically ill infants with bronchiolitis
}

\author{
Luregn J. Schlapbach ${ }^{1,2,3,16}$, Lahn Straney ${ }^{4,16}$, Ben Gelbart ${ }^{5,6}$, \\ Janet Alexander ${ }^{7,8}$, Donna Franklin ${ }^{1}$, John Beca9 , Jennifer A. Whitty ${ }^{10,11}$, \\ Subodh Ganu ${ }^{12,13}$, Barry Wilkins ${ }^{14}$, Anthony Slater ${ }^{2}$, Elizabeth Croston ${ }^{15}$, \\ Simon Erickson ${ }^{15}$ and Andreas Schibler ${ }^{1,2}$ on behalf of the Australian \& New \\ Zealand Intensive Care Society (ANZICS) Centre for Outcomes \& Resource \\ Evaluation (CORE) and the Australian \& New Zealand Intensive Care Society \\ (ANZICS) Paediatric Study Group
}

\begin{abstract}
Affiliations: ${ }^{1}$ Paediatric Critical Care Research Group, Mater Research Institute, The University of Queensland, Brisbane, Australia. ${ }^{2}$ Paediatric Intensive Care Unit, Lady Cilento Children's Hospital, Brisbane, Australia. ${ }^{3}$ Dept of Pediatrics, Inselspital, Bern University Hospital, University of Bern, Bern, Switzerland. ${ }^{4}$ Dept of Epidemiology and Preventive Medicine, Monash University, Melbourne, Australia. ${ }^{5}$ Paediatric Intensive Care Unit, The Royal Children's Hospital, Melbourne, Australia. 'Melbourne Children's Research Institute, Melbourne, Australia. ${ }^{7}$ Australian and New Zealand Paediatric Intensive Care Registry, CORE, Lady Cilento Children's Hospital Brisbane, Brisbane, Australia. ${ }^{8}$ School of Medicine, The University of Queensland, Brisbane, Australia. ${ }^{9}$ Paediatric Intensive Care Unit, Starship Children's Hospital, Auckland, New Zealand. ${ }^{10}$ School of Pharmacy, The University of Queensland, Brisbane, Australia. ${ }^{11} \mathrm{Health}$ Economics Group, Norwich Medical School, University of East Anglia, Norwich, UK. ${ }^{12}$ University of Adelaide, Adelaide, Australia.

${ }^{13}$ Paediatric Intensive Care Unit, Women's and Children's Hospital, Adelaide, Australia. ${ }^{14}$ Paediatric Intensive Care Unit, Children's Hospital Westmead, Sydney, Australia. ${ }^{15}$ Paediatric Intensive Care Unit, Princess Margaret Hospital for Children, Perth, Australia. ${ }^{16}$ L.J. Schlapbach and L. Straney contributed equally to this manuscript.
\end{abstract}

Correspondence: Luregn J. Schlapbach, Paediatric Critical Care Research Group, Mater Research Institute, The University of Queensland, Paediatric Intensive Care Unit, Lady Cilento Children's Hospital, South Brisbane QLD 4101, Australia. E-mail: I.schlapbachduq.edu.au

@ERSpublications

Changing thresholds to admit bronchiolitis patients to PICU have had a major impact on cost and resource utilisation http://ow.ly/AVA630a08rx

Cite this article as: Schlapbach LJ, Straney L, Gelbart B, et al. Burden of disease and change in practice in critically ill infants with bronchiolitis. Eur Respir J 2017; 49: 1601648 [https://doi.org/10.1183/ 13993003.01648-2016].

ABSTRACT Bronchiolitis represents the most common cause of non-elective admission to paediatric intensive care units (ICUs).

We assessed changes in admission rate, respiratory support, and outcomes of infants $<24$ months with bronchiolitis admitted to ICU between 2002 and 2014 in Australia and New Zealand.

During the study period, bronchiolitis was responsible for 9628 (27.6\%) of 34829 non-elective ICU admissions. The estimated population-based ICU admission rate due to bronchiolitis increased by 11.76 per 100000 each year (95\% CI 8.11-15.41). The proportion of bronchiolitis patients requiring intubation decreased from $36.8 \%$ in 2002, to $10.8 \%$ in 2014 (adjusted OR $0.35,95 \%$ CI $0.27-0.46$ ), whilst a dramatic increase in high-flow nasal cannula therapy use to $72.6 \%$ was observed $(p<0.001)$. We observed considerable variability in practice between units, with six-fold differences in risk-adjusted intubation rates that were not explained by ICU type, size, or major patient factors. Annual direct hospitalisation costs due to severe bronchiolitis increased to over USD30 million in 2014.

We observed an increasing healthcare burden due to severe bronchiolitis, with a major change in practice in the management from invasive to non-invasive support that suggests thresholds to admittance of bronchiolitis patients to ICU have changed. Future studies should assess strategies for management of bronchiolitis outside ICUs. 


\section{Introduction}

Bronchiolitis is a common viral lower respiratory tract infection in infants characterised by acute small airway inflammation, and represents the leading cause for hospital admission during the first year of life [1]. In high-income countries, approximately one out of eight infants hospitalised with bronchiolitis requires admission to intensive care units (ICUs) for respiratory support as a result of progressive respiratory distress with respiratory failure and hypoxaemia [2,3]. Despite a general trend towards a reduction in hospital admissions overall, bronchiolitis-related hospitalisation costs have recently increased, amounting to USD1.73 billion per year in the USA [4]. In the past decades, pharmacological interventions have failed to show any benefit and, as a result, consensus guidelines emphasise supportive treatment options [2, 3, 5-8]. While invasive ventilation was traditionally considered to be the cornerstone of treatment for severe bronchiolitis in ICU, over recent years, an increasing number of single-centre studies have reported benefits associated with early use of noninvasive ventilation (NIV) and high-flow nasal cannula (HFNC) therapy to reduce the need for intubation and invasive ventilation in bronchiolitis [9-13].

The aims of this study were to describe the population-based admission rate and severity of bronchiolitis in infants in Australia and New Zealand admitted to intensive care, to determine risk factors for invasive ventilation, and to assess trends in admission rate, management, outcome and associated direct health-care costs over a 13 year period from 2002-2014.

\section{Methods}

Further information regarding the study methods can be found in the supplementary material. A multicentre, binational, retrospective study of all patients reported to the Australian and New Zealand Paediatric Intensive Care (ANZPIC) Registry [14]. The study was approved by the Human Research and Ethics Committee (Mater Health Services HREC, Brisbane, Australia) including waiver of informed consent. The ANZPIC Registry prospectively records demographics, physiological variables at admission, intensive care support, diagnoses and outcomes of paediatric ICU and general ICU admissions in children $<16$ years of age in Australia and New Zealand [14], and captures 92-94\% of all paediatric ICU admissions.

\section{Inclusion and exclusion criteria}

Infants aged <729 days who were admitted with a diagnosis of bronchiolitis [8] and admitted to a paediatric ICU (PICU) or a general ICU in Australia or New Zealand between January 1, 2002 and December 31, 2014 were included. Elective admissions and infants with pre-existing tracheostomies were excluded.

\section{Outcomes and definitions}

The primary outcome was defined as the proportion of infants requiring intubation and invasive ventilation. NIV was defined as continuous positive airway pressure (CPAP) with or without pressure support delivered through a nasal mask, full-face mask, or a nasopharyngeal tube. Mechanical ventilation was defined as either invasive ventilation and/or NIV. Since 2010, the ANZPIC registry has been prospectively recording the use of HFNC oxygen therapy. HFNC was defined as $>1 \mathrm{~L} \cdot \mathrm{kg}^{-1} \cdot \mathrm{min}^{-1}$ flow of a gas oxygen mixture through nasal cannula, and coded separately from mechanical ventilation support [12, 13]. Data analyses were therefore separated into two periods: a period before widespread use of HFNC therapy (2002-2009) and a period after widespread introduction of HFNC therapy (2010-2014). With the exception of one paediatric ICU, HFNC was not routinely used in the main paediatric ICUs and ICUs prior to 2010.

Cost estimate methodology is provided in the supplementary material.

\section{Statistics}

Data are presented as percentages and numbers or means with standard deviations. $t$ tests were used to compare subgroups. Population-based admission rate estimates were calculated. We assessed linear trends in respiratory support over the 13-year period. In addition, trends during the 13 -year study period were assessed by comparing risk-adjusted need for invasive ventilation. We constructed a multivariate

This article has supplementary material available from erj.ersjournals.com

Received: Aug 182016 | Accepted after revision: March 032017

Support statement: The National Medical Health and Research Council (NHMRC) Australian Resuscitation Outcomes Consortium (Aus-ROC) Centre of Research Excellence, who funded Dr Straney, had no involvement in the design, analysis, and writing of the present manuscript. Funding information for this article has been deposited with the Crossref Funder Registry.

Conflict of interest: None declared. 
prediction model for the need for invasive ventilation. For multivariable models, all significant predictors from the univariable analyses were used. We used a backward stepwise elimination procedure to eliminate nonsignificant predictors based on $\mathrm{p}>0.05$.

All paediatric ICUs in Australia and New Zealand contributed to the ANZPIC registry for the entire duration of the study period. The number of general ICUs contributing to the registry increased from six to 19 during the study period. In order to account for potential reporting bias, the following predefined subgroup analyses were performed: 1) specialised paediatric ICUs; 2 ) general (mixed adult and paediatric) ICUs; and 3) paediatric and general ICUs that had contributed to the ANZPIC registry for the entire length of the study period.

Further details of risk prediction models are provided in the supplementary material.

All analyses were conducted by using Stata (version 12.1, Stata Corp, College Station, Texas, USA). p-values of less than 0.05 were considered significant.

\section{Results}

During the study period, bronchiolitis was the most common cause of ICU admission, and was responsible for $9628(27.6 \%)$ of 34829 non-elective admissions in infants below 2 years of age. 324 infants with tracheostomies in situ at time of admission were excluded. Prematurity (20\%), chronic respiratory conditions (10\%) and congenital cardiac disease (7\%) were the most common underlying conditions (table 1). During 2010-2014 (after widespread introduction of HFNC), 5670 infants were admitted with bronchiolitis in comparison to 3634 during 2002-2009 (table 2). In recent years, infants with bronchiolitis admitted to

TABLE 1 Baseline and severity characteristics of patients with bronchiolitis 2002-2014

\begin{tabular}{|c|c|c|c|}
\hline & \multicolumn{2}{|c|}{ Bronchiolitis } & \multirow[t]{2}{*}{ p-value } \\
\hline & 2002-2009 & $2010-2014$ & \\
\hline Total $\mathbf{n}$ & $3634(100 \%)$ & $5670(100 \%)$ & \\
\hline Age days & $91(45-201)$ & $139(57-281)$ & $<0.001$ \\
\hline Neonates $<28$ days & $392(10.8 \%)$ & $463(8.2 \%)$ & $<0.001$ \\
\hline $28-90$ days & $1397(38.4 \%)$ & $1680(29.6 \%)$ & \\
\hline 91 days to 1 years & $1558(42.9 \%)$ & $2784(49.1 \%)$ & \\
\hline $1-2$ years & $287(7.9 \%)$ & $743(13.1 \%)$ & \\
\hline Males & $2128(58.6 \%)$ & $3409(60.1 \%)$ & 0.133 \\
\hline \multicolumn{4}{|l|}{ Category } \\
\hline Admitted to specialist paediatric ICU & $2980(82.0 \%)$ & $3652(64.4 \%)$ & $<0.001$ \\
\hline Interhospital transport & $1667(45.9 \%)$ & $2209(39.0 \%)$ & $<0.001$ \\
\hline \multicolumn{4}{|l|}{ Risk category } \\
\hline Prematurity & $747(20.6 \%)$ & $950(16.8 \%)$ & $<0.001$ \\
\hline Chronic lung disease & $229(6.3 \%)$ & $224(4.0 \%)$ & $<0.001$ \\
\hline Other chronic respiratory disease & $117(3.2 \%)$ & $200(3.5 \%)$ & 0.425 \\
\hline Congenital heart disease & $281(7.7 \%)$ & $330(5.8 \%)$ & $<0.001$ \\
\hline Chronic neurological disease & $77(2.1 \%)$ & $107(1.9 \%)$ & 0.433 \\
\hline Any comorbidity & $1328(36.5 \%)$ & $1640(28.9 \%)$ & $<0.001$ \\
\hline \multicolumn{4}{|l|}{ Aetiology } \\
\hline RSV & $1624(44.7 \%)$ & $2039(36.0 \%)$ & $<0.001$ \\
\hline Influenza & $61(1.7 \%)$ & $84(1.5 \%)$ & 0.454 \\
\hline Human metapneumovirus & $0(0.0 \%)$ & $120(2.1 \%)$ & $<0.001$ \\
\hline Parainfluenzavirus & $67(1.8 \%)$ & $115(2.0 \%)$ & 0.531 \\
\hline Adenovirus & $41(1.1 \%)$ & $192(3.4 \%)$ & $<0.001$ \\
\hline \multicolumn{4}{|l|}{ Severity } \\
\hline Mean paediatric ICU length of stay days & $3.69 \pm 5.82$ & $3.18 \pm 4.33$ & $<0.001$ \\
\hline Mean hospital length of stay days & $12.47 \pm 36.21$ & $9.13 \pm 23.79$ & $<0.001$ \\
\hline PIM2 (mean probability of death) & $0.96 \pm 1.7 \%$ & $0.69 \pm 1.9 \%$ & $<0.001$ \\
\hline Median paediatric ICU length of stay days & $2.29(1.08-4.46)$ & $2.25(1.33-3.75)$ & 0.623 \\
\hline Median hospital length of stay days & $6.93(4.33-11.47)$ & $5.05(3.43-8.25)$ & $<0.001$ \\
\hline PIM2 (median probability of death) & $0.59 \%(0.21-1.00 \%)$ & $0.27 \%(0.18-0.77 \%)$ & $<0.001$ \\
\hline Death & $25(0.7 \%)$ & $10(0.2 \%)$ & $<0.001$ \\
\hline
\end{tabular}

Data are presented as median (interquartile range) or mean $\pm \mathrm{SD}$, unless otherwise stated. ICU: intensive care unit; RSV: respiratory syncytial virus; PIM2: Paediatric Index of Mortality-2. \#: specialist paediatric ICU versus general ICU. 


\begin{tabular}{|c|c|c|c|c|c|c|c|c|c|c|c|}
\hline \multirow[t]{2}{*}{ Year } & \multicolumn{2}{|c|}{ Sites } & \multicolumn{2}{|c|}{$\begin{array}{l}\text { Total ICU admissions due to } \\
\text { respiratory infection }\end{array}$} & \multicolumn{5}{|c|}{$\begin{array}{l}\text { Bronchiolitis admissions rate per } 100000 \\
\text { population }<24 \text { months }\end{array}$} & \multicolumn{2}{|c|}{$\begin{array}{l}\text { Proportion of admitted } \\
\text { patients }\end{array}$} \\
\hline & $\begin{array}{l}\text { Paediatric } \\
\text { ICU }\end{array}$ & General & All & $\begin{array}{l}\text { Paediatric } \\
\text { ICU }\end{array}$ & All & $\begin{array}{l}\text { Paediatric } \\
\text { ICU }\end{array}$ & General & 13-year" & $\begin{array}{l}\text { General/all } \\
\text { (n/n } \%)\end{array}$ & All & Paediatric \\
\hline 2002 & 9 & 6 & 589 & 549 & 62.5 (383) & 58.5 (358) & $4.1(25)$ & 62.0 (380) & $6.5 \%$ & $19.8 \%$ & $19.5 \%$ \\
\hline 2003 & 9 & 7 & 575 & 508 & 51.9 (317) & $43.6(266)$ & 8.4 (51) & 49.3 (301) & $16.1 \%$ & $16.7 \%$ & $15.3 \%$ \\
\hline 2004 & 9 & 7 & 629 & 583 & $59.4(366)$ & 55.0 (339) & $4.4(27)$ & 58.1 (358) & $7.4 \%$ & $17.2 \%$ & $17.0 \%$ \\
\hline 2005 & 9 & 7 & 640 & 568 & 60.7 (380) & 52.4 (328) & $8.3(52)$ & 56.5 (354) & $13.7 \%$ & $19.4 \%$ & $18.1 \%$ \\
\hline 2006 & 9 & 8 & 780 & 637 & 82.7 (531) & 66.5 (427) & $16.2(104)$ & $72.0(462)$ & $19.6 \%$ & $24.3 \%$ & $22.2 \%$ \\
\hline 2007 & 9 & 12 & 824 & 641 & 78.3 (529) & 59.7 (403) & $18.7(126)$ & $62.2(420)$ & $23.8 \%$ & $22.3 \%$ & $19.9 \%$ \\
\hline 2008 & 9 & 13 & 921 & 747 & 82.5 (579) & $63.5(446)$ & 18.9 (133) & $64.2(451)$ & $23.0 \%$ & $23.3 \%$ & $21.1 \%$ \\
\hline 2009 & 9 & 15 & 891 & 712 & 76.8 (549) & 57.8 (413) & $19.0(136)$ & 62.0 (443) & $24.8 \%$ & $22.9 \%$ & $20.0 \%$ \\
\hline 2010 & 9 & 15 & 1083 & 811 & $106.5(766)$ & 76.5 (550) & $30.0(216)$ & 78.8 (567) & $28.2 \%$ & $26.7 \%$ & $23.3 \%$ \\
\hline 2011 & 9 & 15 & 1308 & 897 & $123.5(880)$ & 80.0 (570) & 43.5 (310) & 85.5 (609) & $35.2 \%$ & $27.8 \%$ & $23.0 \%$ \\
\hline 2012 & 9 & 17 & 1606 & 1098 & $167.0(1206)$ & 108.0 (780) & $59.0(426)$ & 114.5 (827) & $35.3 \%$ & $34.4 \%$ & $29.0 \%$ \\
\hline 2013 & 9 & 19 & 1739 & 1189 & 174.5 (1290) & 112.9 (835) & 61.5 (455) & 120.0 (887) & $35.3 \%$ & $33.6 \%$ & $28.3 \%$ \\
\hline 2014 & $10^{\pi}$ & 19 & 1969 & 1265 & 208.9 (1528) & 125.3 (917) & $83.5(611)$ & $138.3(1012)$ & $40.0 \%$ & $37.1 \%$ & $30.7 \%$ \\
\hline B (95\% CI) & & & $\begin{array}{c}113.65 \\
(86.99-140.32)\end{array}$ & $\begin{array}{c}61.36 \\
(46.65-76.1)\end{array}$ & $\begin{array}{c}11.84 \\
(8.24- \\
15.45]\end{array}$ & $\begin{array}{l}5.83 \\
(3.75- \\
7.91)\end{array}$ & $\begin{array}{l}6.01 \\
(4.37- \\
7.65)\end{array}$ & $\begin{array}{l}6.25 \\
(3.86-8.63)\end{array}$ & $\begin{array}{c}0.03 \\
(0.02-0.03)\end{array}$ & $\begin{array}{c}0.02 \\
(0.01-0.02)\end{array}$ & $\begin{array}{c}0.01 \\
(0.01-0.01)\end{array}$ \\
\hline $\begin{array}{l}\mathrm{p} \text {-value (for } \\
\text { trend) }\end{array}$ & & & $<0.0001$ & $<0.0001$ & $<0.0001$ & 0.0001 & $<0.0001$ & 0.0001 & $<0.0001$ & $<0.0001$ & $<0.0001$ \\
\hline
\end{tabular}

ICU: intensive care unit. "\# dataset restricted to units that contributed data to the registry for the entire study period 2002-2014; ": two paediatric ICUs that had contributed data to the registry since 2002 merged at the end of 2014 to one new facility. 
ICU were older, less likely to require interhospital transfer, more likely to be admitted to a general ICU, and less likely to have underlying diseases $(\mathrm{p}<0.001)$. The average severity of disease as measured by mean probability of death (PIM2) decreased significantly, and ICU and hospital length of stay (LOS) decreased accordingly. The crude mortality over the entire study period was $0.38 \%$ (35/9304). The re-calibrated PIM2 standardised mortality ratio declined from $1.53(0.99-2.26)$ in $2002-2009$ to $0.54(0.26-0.98)$ in 2010-2014.

The annual number of infants with bronchiolitis admitted to ICU (including paediatric and general ICUs) increased from 383 cases in 2002 to 1528 cases in 2014 (table 2, figure 1). The total number of all non-elective ICU admissions per year during this time increased from 1933 to 4115. The estimated population-based age-standardised admission rate of bronchiolitis increased during the study period with an average annual increase of 11.76 per 100000 infants <24 months (95\% CI 8.11-15.41). The change in admission rate was most pronounced in general ICUs, which took $40 \%$ of all bronchiolitis admissions requiring intensive care in $2014(\mathrm{p}<0.0001)$. The increase in admission was less marked when restricting analyses to paediatric ICUs (annual increase 5.80 per $100000,95 \%$ CI 3.68-7.90), and to ICUs that had contributed to the registry for the entire study duration (6.21 per $100000,95 \%$ CI $3.80-8.63)$. The increase in bronchiolitis admission was higher compared to admissions due to any other respiratory infection, and higher compared to all annual paediatric ICU admissions, which increased from 1933 to 4115 (table 2).

In $2002,37 \%$ of patients with bronchiolitis were intubated/invasively ventilated, in comparison to only $11 \%$ in 2014 ( $\mathrm{p}<0.001$, figure 2 and supplementary table S1). In view of the concomitant dramatic increase in bronchiolitis-related ICU admission rates, we calculated estimates of population-based intubation rates (supplementary table S2). Absolute intubation numbers and population-based intubation rates in infants with bronchiolitis did not decrease significantly during the study period $(p>0.05)$. We observed a decrease in the proportion of intubations performed in ICU after the first hour of admission from 66.2\% (674/ 1018 ) in $2002-2009$ to $43.6 \%$ (394/903) in 2010-2014. The time to intubation in infants that were not intubated within the first hour of admission did not change during the study period $(\mathrm{p}=0.840$; supplementary figure $\mathrm{S} 1$ ). Over the same period, the length of mechanical ventilation (invasive ventilation and/or NIV) decreased significantly from a mean duration of $109 \mathrm{~h}$ to $69 \mathrm{~h}$ (average decrease $3.4 \mathrm{~h}$ per mechanically ventilated patient per year; 95\% CI 2.25-4.56). Following the introduction of HFNC therapy in most paediatric ICUs, the use of HFNC in infants with bronchiolitis increased over 2010-2014 to

a)

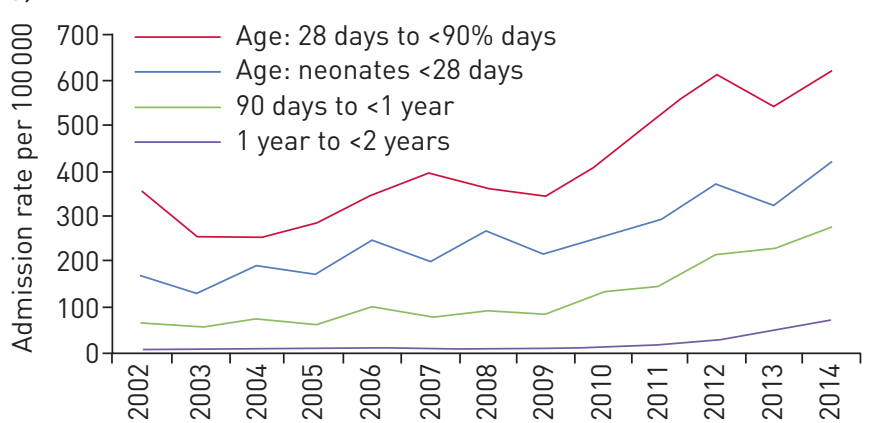

c)

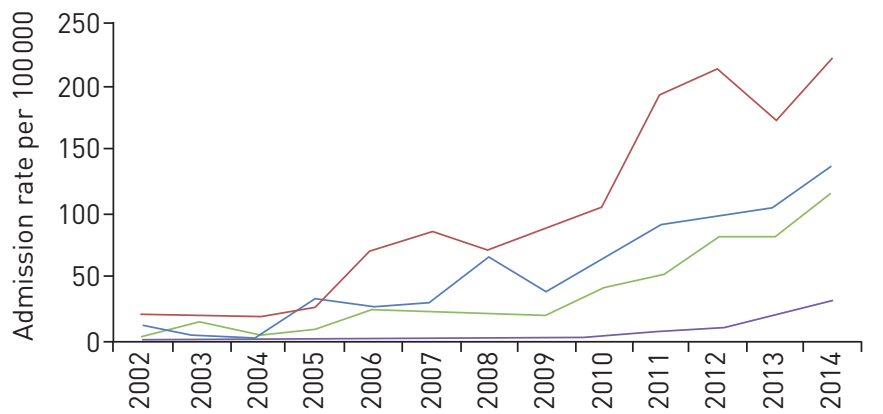

b)

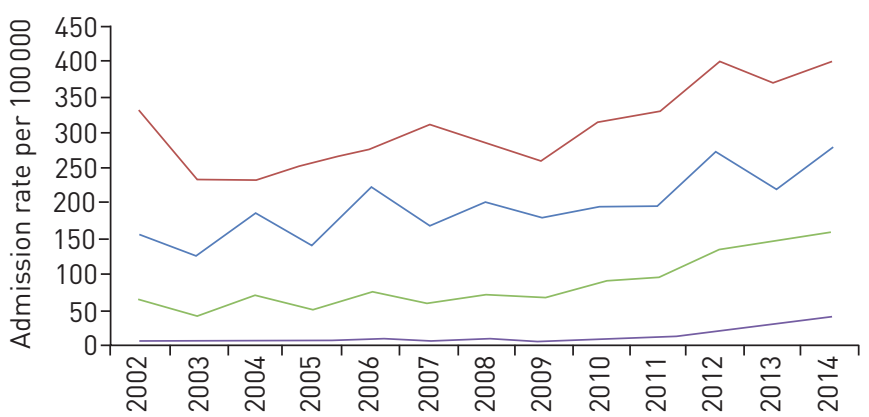

d)

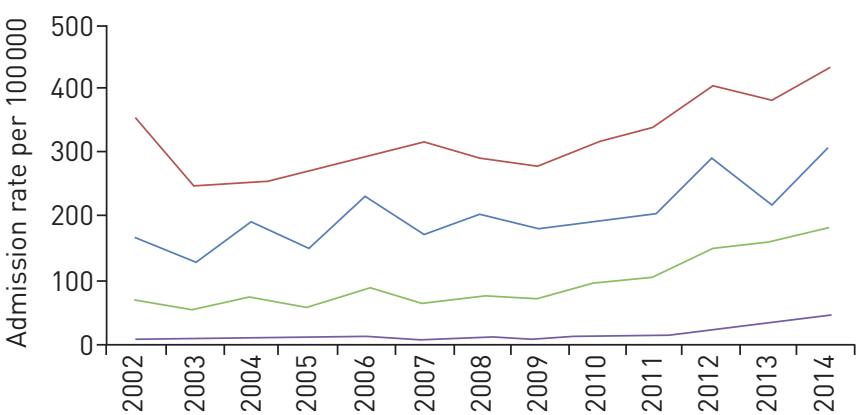

FIGURE 1 Estimated population-based intensive care unit (ICU) admission rates due to bronchiolitis. ICU admission rates per 100000 infants $<24$ months of age and year are shown for al all ICU admissions captured in the Australian and New Zealand Paediatric Intensive Care Registry; b) admissions to paediatric PICUs; c) admissions to general Intensive Care Units (ICUs); and d) admissions to paediatric ICUs and ICUs that contributed to the registry for the entire study duration 2002-2014. 

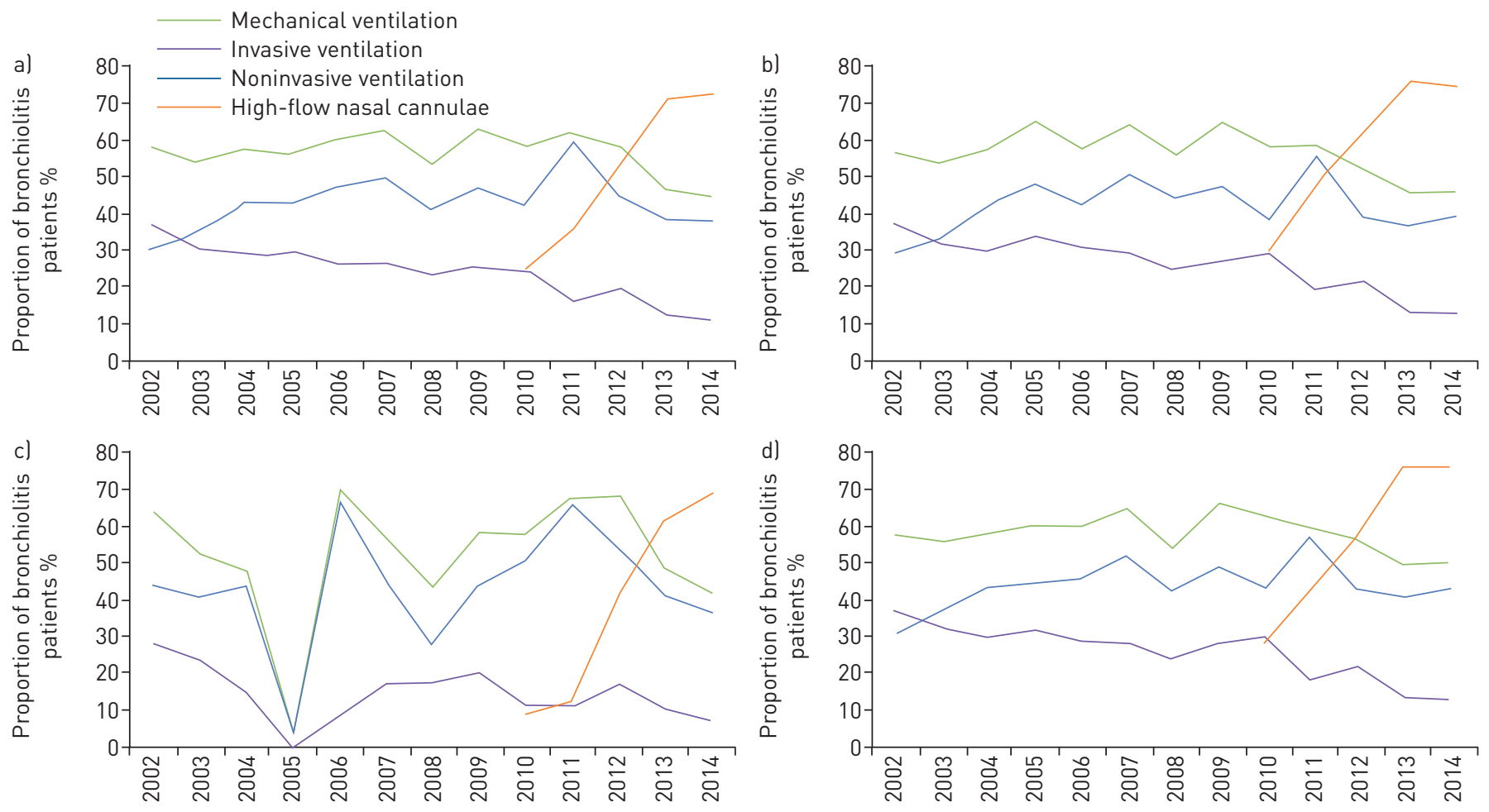

FIGURE 2 Changes in respiratory support mode during the study period 2002-2014 in infants admitted to intensive care unit (ICU) with bronchiolitis. Mechanical ventilation is defined as intubation and/or noninvasive ventilation. Proportions of respiratory support mode used per patient (more than one modality allowed) are shown for a) all ICU admissions captured in the Australian and New Zealand Paediatric Intensive Care Registry; b) admissions to paediatric ICUs; c) admissions to general ICUs; and d) admissions to paediatric ICUs and ICUs that contributed to the registry for the entire study duration 2002-2014.

72.6\%; this was accompanied by a reduction in the use of NIV. The findings observed in the entire cohort were confirmed in subgroup analyses restricted to paediatric ICUs only, and to paediatric ICUs and ICUs that had contributed to the registry for the entire study duration (figure 2 and supplementary tables S1 and S2).

In multivariate analyses, age, interhospital transport, major chronic conditions, prematurity, and severity indicators such as low systolic blood pressure were identified as significant predictors for intubation (table 3; AUC 0.76, 0.75-0.77). Each year was associated with a $0.9 \%$ decline in the risk of intubation

TABLE 3 Multivariate model to prediction of likelihood of requiring intubation and invasive ventilation in critically ill infants with bronchiolitis

\begin{tabular}{lccrrr} 
& Odds ratio & $\mathbf{9 5 \%} \mathbf{C l}$ & $\boldsymbol{\beta}$ & $\mathbf{9 5 \%} \mathbf{C l}$ & $\mathbf{p}$-value \\
\hline Age (days/30) & 0.970 & 0.957 to 0.983 & -0.013 & -0.044 to -0.017 & $<0.001$ \\
Interhospital transport & 2.718 & 2.415 to 3.058 & 0.434 & 0.882 to 1.118 & $<0.001$ \\
Chronic neurological condition & 1.723 & 1.187 to 2.502 & 0.236 & 0.171 to 0.917 & 0.004 \\
Chronic respiratory condition & 1.585 & 1.182 to 2.124 & 0.200 & 0.167 to 0.753 & 0.002 \\
Bronchopulmonary dysplasia & 1.686 & 1.324 to 2.148 & 0.227 & 0.280 to 0.765 & $<0.001$ \\
Congenital heart defect & 1.875 & 1.536 to 2.289 & 0.273 & 0.429 to 0.828 & $<0.001$ \\
Prematurity & 1.320 & 1.147 to 1.520 & 0.121 & 0.137 to 0.419 & $<0.001$ \\
RSV & 1.561 & 1.387 to 1.757 & 0.193 & 0.327 to 0.564 & $<0.001$ \\
Influenza/parainfluenzae & 2.001 & 1.523 to 2.629 & 0.301 & 0.421 to 0.967 & $<0.001$ \\
Systolic blood pressure $\leqslant \mathbf{7 0} \mathbf{m m H g}$ & 4.034 & 3.160 to 5.151 & 0.606 & 1.151 to 1.639 & $<0.001$ \\
Base excess & 0.947 & 0.929 to 0.967 & -0.023 & -0.074 to -0.034 & $<0.001$ \\
(Base excess)^2 & 1.010 & 1.008 to 1.013 & 0.004 & 0.008 to 0.012 & $<0.001$ \\
Constant & 0.087 & 0.063 to 0.121 & -1.060 & -2.768 to -2.113 & $<0.001$ \\
\hline
\end{tabular}

Data are based on a saturated mix-effects logistic regression model clustering on site and adjusted for all variables shown in the table. RSV: respiratory syncytial virus. 

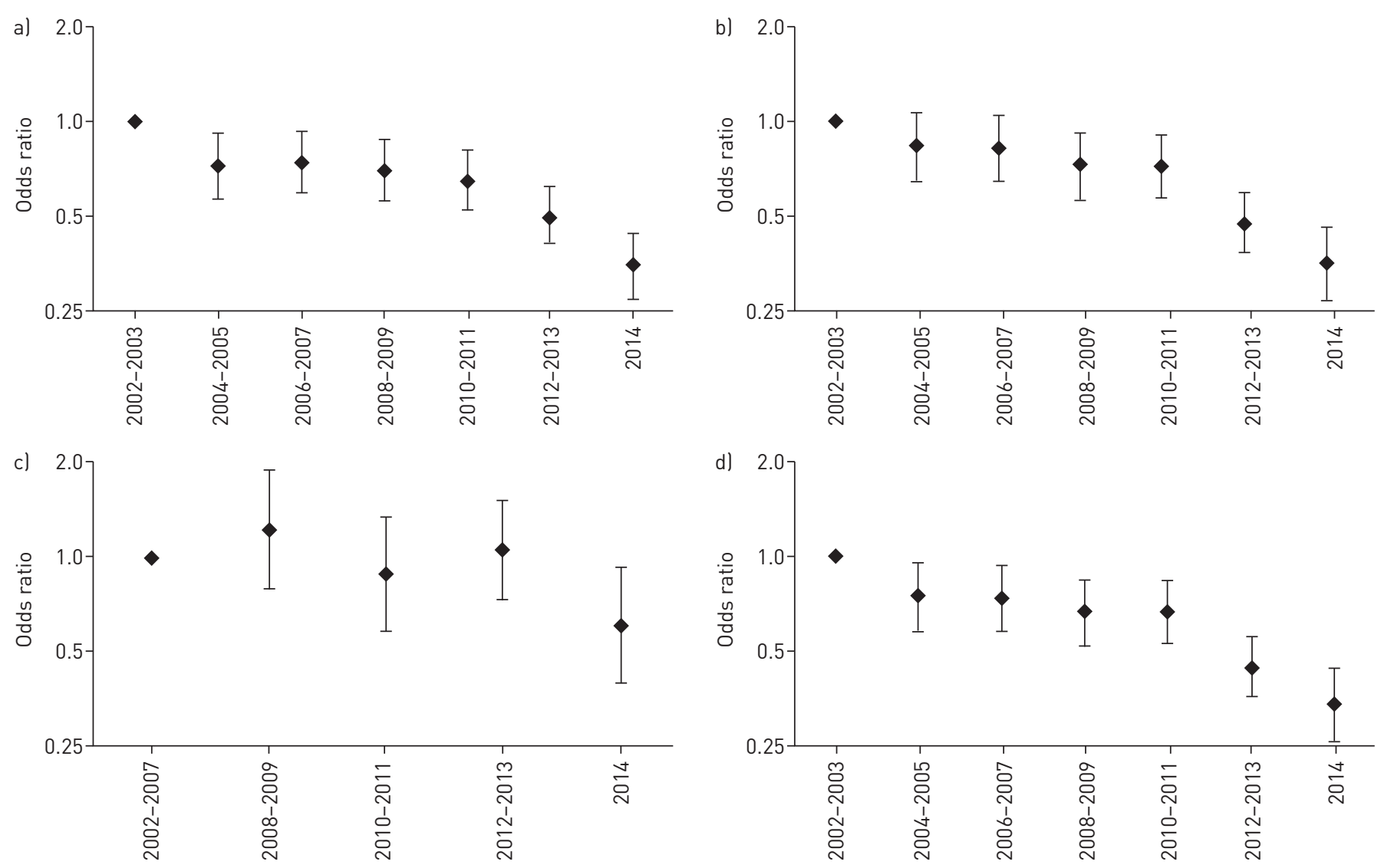

FIGURE 3 Adjusted risk for intubation/invasive ventilation in infants with bronchiolitis during the study period. Odds ratios are adjusted for age, interhospital transport, chronic respiratory and neurological conditions, prematurity, congenital heart disease, specific respiratory viruses, low systolic blood pressure, and are shown for al all intensive care unit (ICU) admissions captured in the Australian and New Zealand Paediatric Intensive Care Registry; b) admissions to paediatric ICUs; c) admissions to general ICUs; and d) admissions to paediatric ICUs and ICUs that contributed to the registry for the entire study duration 2002-2014.

(95\% CI $0.81-1.00 \%$ ). The overall adjusted odds for intubation decreased significantly from 2002/2003 to 2014 (OR 0.35, 95\% CI 0.27-0.44; p<0.001) (figure 3). The model performed comparably when restricted to infants that were not intubated on arrival to paediatric ICU (supplementary table S3A). For children treated with HFNC, younger age, bronchopulmonary dysplasia, congenital heart defects, low blood pressure, and increased negative base excess were the main predictors of intubation $(\mathrm{p}<0.01)$ (supplementary table S3B).

When comparing the risk-adjusted rate of an infant with bronchiolitis receiving invasive ventilation, important differences between units were noted (figure 4). When compared to an intercept-only mixed effects model, a model that controlled for year and patient risk factors (table 3) demonstrated a 39.1\% reduction in the variance of the random effect. This difference suggests that $60.9 \%$ of the variation in

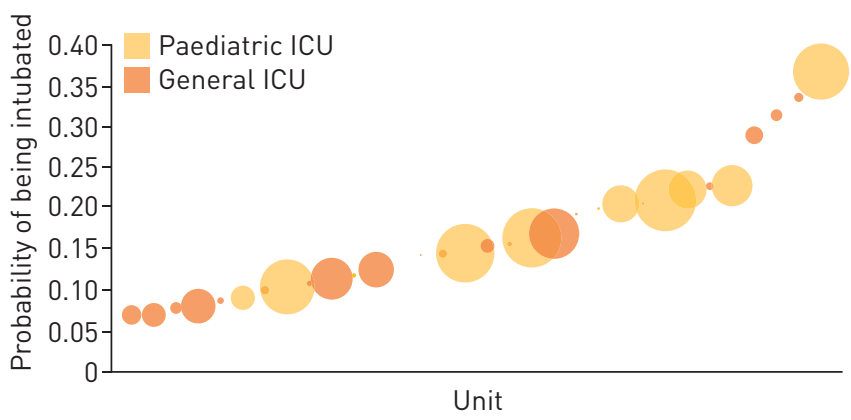

FIGURE 4 Comparison of the probability of an infant with bronchiolitis receiving invasive ventilation between different paediatric and general intensive care units (ICUs). Area of bubble indicates relative admission numbers. 


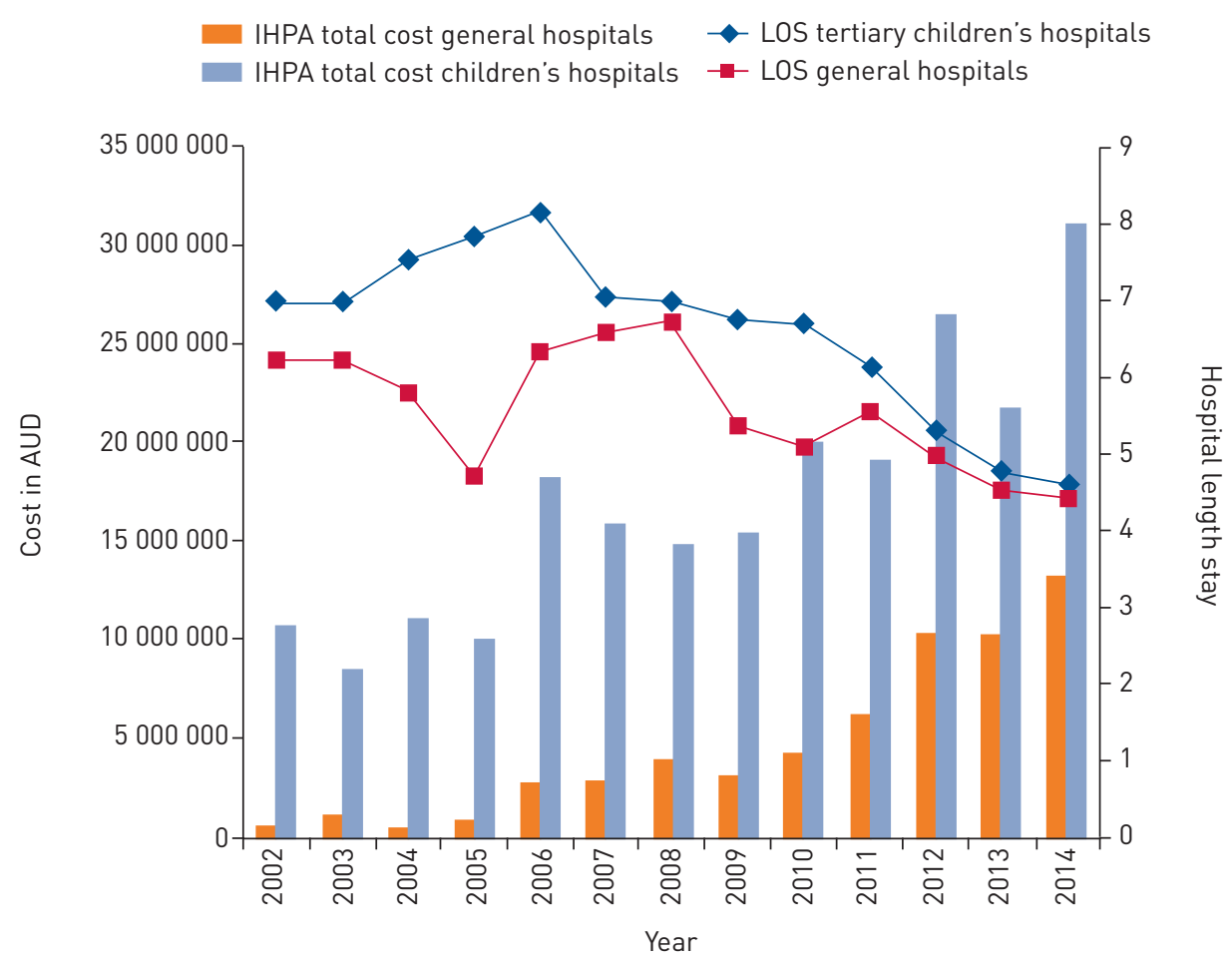

FIGURE 5 Annual direct hospitalisation cost (in AUD 2014) due to severe bronchiolitis requiring intensive care unit admission in Australia and New Zealand, 2002-2014. Length of stay (LOS), and associated costs estimated by Independent Hospital Pricing Authority (IHPE) Efficient Price Determination are shown for children`s hospitals and general hospitals.

intubation rates were neither explained by the case mix nor by time trends, and likely reflect underlying differences in unit-to-unit practice. Using the average risk profile for intubation, the probability of a child with bronchiolitis being intubated varied from $6.9 \%$ to $36.7 \%$ across units.

We assessed total direct hospitalisation-related costs in infants requiring ICU admission, including ICU and ward costs. Although a reduction in LOS over the period of 2002-2014 occurred, the total annual costs increased from AUD11.4 million in 2002 to \$44.3 million in 2014 (supplementary table S4 and figure 5). The total costs related to bronchiolitis admitted to specialised paediatric ICUs increased from $\$ 10.7$ million to $\$ 31.1$ million over the same period.

\section{Discussion}

This large binational study, including over 9000 critically ill infants with bronchiolitis, demonstrates that severe bronchiolitis is responsible for a huge burden of disease, resulting in over USD30 million direct costs each year in Australia and New Zealand. Bronchiolitis has a major impact on ICU resource consumption, accounting for $>25 \%$ of non-elective PICU admissions in infants. In the absence of high-grade evidence for treatment of bronchiolitis in ICU, we observed a dramatic increase in the number of children admitted to intensive care in Australia and New Zealand with bronchiolitis, concomitant with the increased use of HFNC therapy. In view of close to zero mortality in infants with severe bronchiolitis, these findings indicate an urgent need for future studies investigating whether a proportion of these patients may be safely managed outside the ICU, hence reducing associated healthcare costs.

By estimating population-based ICU admission rates, we were able to document a major change in practice over the past decade, with a dramatic increase in ICU admissions in infants with bronchiolitis that do not require invasive ventilation. The increase observed in general ICUs may have been enhanced by reporting bias, as more general ICUs have contributed data to the study database over recent years. However, the findings were reproduced in subgroup analyses restricted to paediatric ICUs, and restricted to those centres that had provided data for the entire study duration. Infants with bronchiolitis admitted in recent years to ICU were less likely to have underlying comorbidities, were older, and their predicted mortality was lower, which suggests changing thresholds for ICU admission. At the same time, we observed a considerable variability in practice between units, with over six-fold difference in risk-adjusted intubation rates that was not explained by ICU type, size, nor major patient factors. Our findings support 
a recent North American study on bronchiolitis that observed $>3.5$-fold variation in the risk of intubation between paediatric ICUs, and a high variability in the proportion of patients exposed to other non-evidence-based interventions [15]. While bronchiolitis hospital admission rates and length of stay have been proposed as benchmarks in the NHS Atlas of Variation in Healthcare (www.chimat.org.uk/ variation), ICU resource use has not been investigated. Notably, the study period witnessed the implementation of rapid response teams and early warning tools (EWTs) in the major paediatric hospitals, followed by regional hospitals. While EWTs have been shown to predict the need for ICU admission, and reduce cardiopulmonary arrests in the ward setting [16], to the best of our knowledge they have not been validated specifically for bronchiolitis. Indeed, infants with severe bronchiolitis commonly manifest tachycardia, tachypnoea, and increased work of breathing, and may trigger EWTs relatively rapidly, with an unknown impact on health outcomes and resource utilisation.

Our data show that this increase in ICU admission rates was associated with a rapid expansion in costs, despite a reduction in LOS in intensive care and a shorter duration of ventilation. Our costs are comparable to those in a recent European study, which reported a four times higher cost for children requiring ICU compared to those treated on the ward (EUR8061 versus EUR1834) [17].

Our study demonstrates that, despite lack of larger trials on HFNC use in infants, HFNC therapy has become the most common support mode for critically ill infants with bronchiolitis in Australia and New Zealand recently, with over 70\% of admissions in 2014 supported with HFNC. At the same time, while the proportion of admissions requiring intubation and invasive ventilation dropped, we did not observe a significant reduction in the absolute number of children requiring invasive ventilation. Our observation parallels those of several single-centre studies that reported a drop in intubation rates in patients with severe bronchiolitis following the increased use of NIV and HFNC [10,12, 13, 18-22], and suggests that these have led to a major change of practice in the absence of higher grade evidence. Recent large randomised trials in adults have demonstrated efficacy of HFNC therapy for acute hypoxemic respiratory failure $[23,24]$. In the neonatal population, HFNC therapy was shown to be non-inferior to nasal CPAP $[25,26]$. While the physiological effects of HFNC therapy, including provision of $4-5 \mathrm{cmH}_{2} \mathrm{O} C P A P$, reduction in inspiratory work of breathing through provision of flows matching or greater than peak inspiratory flows, and washout of nasopharyngeal dead space have been independently confirmed [27, 28], there is a lack of randomised controlled trials to support either outcome benefit or cost benefit of HFNC therapy in the paediatric age group. A recent adult single-centre study reported higher mortality in patients treated with HFNC that required delayed emergency intubation [29]. In our cohort, the increasing use of HFNC was not associated with a delay in intubations, and in fact the proportions of late intubations, mortality, length of mechanical ventilation, and length of ICU stay decreased.

Advantages of HFNC therapy include an excellent safety profile, low equipment costs, easy application, and increased patient comfort compared to other forms of respiratory support. As a result, the application of HFNC therapy outside ICU settings has been increasingly tested, and single-centre studies suggest safety, feasibility, and efficiency of such an approach [18], although the translation of this approach into better outcomes remains to be proven. Bronchiolitis is a relatively uniform disease with close to zero mortality, patients mostly demonstrate gradual rather than precipitous deterioration, and predictors can be used to stratify severity [30]. As a result, this disease may in fact be optimally suited to design interventional trials aiming to reduce the number of intensive care admissions [31].

We believe the results of this study have several implications for the design of further research on bronchiolitis. First, they highlight an urgent need to validate early warning tools for bronchiolitis, as these may have a direct impact on intensive care admission rates. Secondly, improved markers of severity, including viral genomic load [32], may assist in optimising risk stratification to the target groups most likely to develop severe respiratory failure. Thirdly, the variation in respiratory support practice suggests a lack of standardised protocols $[5,8]$. Fourthly, the dramatic change of practice seen in respiratory support modes warrants a paediatric ICU trial of HFNC therapy versus NIV versus low-flow oxygen for infants and children with respiratory failure [24]. Finally, our data suggest that it is likely that a considerable proportion of intensive care patients may be good candidates to be considered for treatment outside ICU; whether HFNC therapy provides a cost benefit in such a setting has to be tested by large trials [31]. Importantly, trials on optimal respiratory support outside ICU may inform on the development and implementation of cost-effective interventions relevant at a global scale, with the potential to translate into mortality benefits in resource-poor countries [33].

Several limitations of this study need to be considered. While we were not able to assess overall hospital admissions due to bronchiolitis, we captured every patient admitted to a general or paediatric ICU in Australia and New Zealand providing data to the registry. Although bronchiolitis is well-defined as a clinical entity [8], overlap with reactive airway diseases may lead to diagnostic challenges, potentially 
resulting in overdiagnosis of the disease. The use of HFNC was not prospectively captured prior to 2010, and we therefore cannot comment on how frequently HFNC was used as a respiratory support mode prior to 2010. Furthermore, we did not have access to detailed microbiological data, and extended nasopharyngeal virus polymerase chain reaction tests were not consistently performed in centres throughout the study. Because the large majority of infants with bronchiolitis admitted to ICU did not receive intra-arterial monitoring on admission, we were unable to stratify by the degree of hypoxaemia. Finally, inspired oxygen fraction $\left(\mathrm{FIO}_{2}\right)$ to arterial oxygen saturation measured by pulse oximetry ratios could not be used as the true $\mathrm{FIO}_{2}$ is unknown in HFNC or low flow oxygen therapy. In addition, we did not have access to carbon dioxide tension values measured on admission to ICU; however, elevated carbon dioxide tension values represent a common finding in bronchiolitis and may not necessarily indicate a need for intubation [34].

In conclusion, severe bronchiolitis remains responsible for a huge burden of disease. We observed a major change in practice in the management of severe bronchiolitis with dramatically increased use of HFNC therapy despite the lack of high-grade evidence for treatment benefit in this age group. Our data suggest that thresholds to admit bronchiolitis patients to intensive care have reduced over the past decade, with a major impact on healthcare-related costs and resource utilisation in ICUs in Australia and New Zealand. International trials addressing the risk stratification and safe management of bronchiolitis outside intensive care settings are urgently warranted.

\section{Acknowledgements}

We thank the Paediatric Study Group of the Australian and New Zealand Intensive Care Society for supporting this study (Simon Erickson, Princess Margaret Hospital for Children, Perth, Australia; Andreas Schibler, Anthony Slater, Debbie Long, Jan Alexander, Lady Cilento Children's Hospital, Brisbane, Australia; John Beca, Claire Sherring, Starship Children's Hospital, Auckland, New Zealand; Gary Williams, Janelle Young, Mary Lou Morritt, Sydney Children's Hospital, Randwick, Australia; Johnny Millar, Carmen Del Zoppo, Warwick Butt, Royal Children's Hospital, Melbourne, Australia; Subodh Ganu, Georgia Letton, Women's and Children's Hospital, Adelaide, Australia; Marino Festa, Westmead Children's Hospital, Sydney, Australia). We thank the intensivists, data managers and other staff in the participating ICUs for their data contributions. We thank Peter Hicks, ANZICS CORE, for his help in health economic analyses. The ANZPIC Registry is supported by the Australian and New Zealand Intensive Care Society, the Ministry of Health (New Zealand), and State and Territory Health Departments through the Australian Health Ministers' Advisory Council.

Author contributions are as follows: L.J. Schlapbach and L. Straney had full access to all the data in the study and take responsibility for the integrity of the data and the accuracy of the data analysis. L.J. Schlapbach was responsible for the study design, prepared the first manuscript draft and approved the final version. L. Straney and A. Schibler were involved in study design, analyses, preparation of the first manuscript draft and final version. A. Slater led the design and maintenance of the ANZPIC Registry since inception, was involved in study design, and approved the final version. J.A. Whitty and D. Franklin performed healthcare-cost-related analyses and interpretation. J. Beca, B. Wilkins, S. Erickson, E. Croston, B. Gelbart, S. Ganu and J. Alexander were involved in study design, data collection, and manuscript preparation, and approved the final version.

\section{References}

1 Meissner HC. Viral bronchiolitis in children. N Engl J Med 2016; 374: 62-72.

2 Oakley E, Borland M, Neutze J, et al. Nasogastric hydration versus intravenous hydration for infants with bronchiolitis: a randomised trial. Lancet Respir Med 2013; 1: 113-120.

3 Zorc JJ, Hall CB. Bronchiolitis: recent evidence on diagnosis and management. Pediatrics 2010; 125: 342-349.

4 Hasegawa K, Tsugawa Y, Brown DF, et al. Trends in bronchiolitis hospitalizations in the United States, 20002009. Pediatrics 2013; 132: 28-36.

5 Su SC, Chang AB. Improving the management of children with bronchiolitis: the updated American Academy of Pediatrics Clinical Practice Guideline. Chest 2014; 146: 1428-1430.

6 Cunningham S, Rodriguez A, Adams T, et al. Oxygen saturation targets in infants with bronchiolitis (BIDS): a double-blind, randomised, equivalence trial. Lancet 2015; 386: 1041-1048.

7 Schuh S, Freedman S, Coates A, et al. Effect of oximetry on hospitalization in bronchiolitis: a randomized clinical trial. JAMA 2014; 312: 712-718.

8 Ralston SL, Lieberthal AS, Meissner HC, et al. Clinical practice guideline: the diagnosis, management, and prevention of bronchiolitis. Pediatrics 2014; 134: e1474-e1502.

9 Essouri S, Laurent M, Chevret L, et al. Improved clinical and economic outcomes in severe bronchiolitis with pre-emptive nCPAP ventilatory strategy. Intensive Care Med 2014; 40: 84-91.

10 Ganu SS, Gautam A, Wilkins B, et al. Increase in use of non-invasive ventilation for infants with severe bronchiolitis is associated with decline in intubation rates over a decade. Intensive Care Med 2012; 38: 1177-1183.

11 Lazner MR, Basu AP, Klonin H. Non-invasive ventilation for severe bronchiolitis: analysis and evidence. Pediatr Pulmonol 2012; 47: 909-916.

12 Schibler A, Pham TM, Dunster KR, et al. Reduced intubation rates for infants after introduction of high-flow nasal prong oxygen delivery. Intensive Care Med 2011; 37: 847-852.

13 Schlapbach LJ, Schaefer J, Brady AM, et al. High-flow nasal cannula (HFNC) support in interhospital transport of critically ill children. Intensive Care Med 2014; 40: 592-599.

14 Schlapbach LJ, Straney L, Alexander J, et al. Mortality related to invasive infections, sepsis, and septic shock in critically ill children in Australia and New Zealand, 2002-13: a multicentre retrospective cohort study. Lancet Infect Dis 2015; 15: 46-54. 
15 Carroll CL, Faustino EV, Pinto MG, et al. A regional cohort study of the treatment of critically ill children with bronchiolitis. J Asthma 2016; 53: 1006-1011.

16 Gold DL, Mihalov LK, Cohen DM. Evaluating the Pediatric Early Warning Score (PEWS) system for admitted patients in the pediatric emergency department. Acad Emerg Med 2014; 21: 1249-1256.

17 Heikkila P, Forma L, Korppi M. Hospitalisation costs for infant bronchiolitis are up to 20 times higher if intensive care is needed. Acta Paediatr 2015; 104: 269-273.

18 Mayfield S, Bogossian F, O'Malley L, et al. High-flow nasal cannula oxygen therapy for infants with bronchiolitis: pilot study. J Paediatr Child Health 2014; 50: 373-378.

19 Thorburn K, Ritson P. Heated, humidified high-flow nasal cannula therapy in viral bronchiolitis--Panacea, passing phase, or progress? Pediatr Crit Care Med 2012; 13: 700-701.

20 Arora B, Mahajan P, Zidan MA, et al. Nasopharyngeal airway pressures in bronchiolitis patients treated with high-flow nasal cannula oxygen therapy. Pediatr Emerg Care 2012; 28: 1179-1184.

21 Abboud PA, Roth PJ, Skiles CL, et al. Predictors of failure in infants with viral bronchiolitis treated with high-flow, high-humidity nasal cannula therapy. Pediatr Crit Care Med 2012; 13: e343-e349.

22 McKiernan C, Chua LC, Visintainer PF, et al. High flow nasal cannulae therapy in infants with bronchiolitis. J Pediatr 2010; 156: 634-638.

23 Stephan F, Barrucand B, Petit P, et al. High-flow nasal oxygen vs. noninvasive positive airway pressure in hypoxemic patients after cardiothoracic surgery: a randomized clinical trial. JAMA 2015; 313: 2331-2339.

24 Frat JP, Thille AW, Mercat A, et al. High-flow oxygen through nasal cannula in acute hypoxemic respiratory failure. N Engl J Med 2015; 372: 2185-2196.

25 Manley BJ, Owen LS, Doyle LW, et al. High-flow nasal cannulae in very preterm infants after extubation. $N$ Engl J Med 2013; 369: 1425-1433.

26 Lavizzari A, Colnaghi M, Ciuffini F, et al. Heated, humidified high-flow nasal cannula vs nasal continuous positive airway pressure for respiratory distress syndrome of prematurity: a randomized clinical noninferiority trial. JAMA Pediatr 2016; in press [https://doi.org/10.1001/jamapediatrics.2016.1243].

27 Milesi C, Baleine J, Matecki S, et al. Is treatment with a high flow nasal cannula effective in acute viral bronchiolitis? A physiologic study. Intensive Care Med 2013; 39: 1088-1094.

28 Hough JL, Pham TM, Schibler A. Physiologic effect of high-flow nasal cannula in infants with bronchiolitis. Pediatr Crit Care Med 2014; 15: e214-e219.

29 Kang BJ, Koh Y, Lim CM, et al. Failure of high-flow nasal cannula therapy may delay intubation and increase mortality. Intensive Care Med 2015; 41: 623-632.

30 Hasegawa K, Pate BM, Mansbach JM, et al. Risk factors for requiring intensive care among children admitted to ward with bronchiolitis. Acad Pediatr 2015; 15: 77-81.

31 Franklin D, Dalziel S, Schlapbach LJ, et al. Early high flow nasal cannula therapy in bronchiolitis, a prospective randomised control trial (protocol): A Paediatric Acute Respiratory Intervention Study (PARIS). BMC Pediatr 2015; 15: 183.

32 Hasegawa K, Jartti T, Mansbach JM, et al. Respiratory syncytial virus genomic load and disease severity among children hospitalized with bronchiolitis: multicenter cohort studies in the United States and Finland. J Infect Dis 2015; 211: 1550-1559.

33 Chisti MJ, Salam MA, Smith JH, et al. Bubble continuous positive airway pressure for children with severe pneumonia and hypoxaemia in Bangladesh: an open, randomised controlled trial. Lancet 2015; 386: 1057-1065.

34 Essouri S, Carroll C, Pediatric Acute Lung Injury Consensus Conference G. Noninvasive support and ventilation for pediatric acute respiratory distress syndrome: proceedings from the Pediatric Acute Lung Injury Consensus Conference. Pediatr Crit Care Med 2015; 16: Suppl. 1, S102-S110. 